\title{
MODELAGEM PROBABILÍSTICA DE SETORES DE UM SISTEMA ELÉTRICO
}

\author{
Gauss M. Cordeiro* \\ Cláudio T. Cristino \\ Andrea A. Prudente
}

Departamento de Estatística e Informática (DEINFO)

Universidade Federal Rural de Pernambuco (UFRPE)

Recife - PE, Brasil

gauss@,deinfo.ufrpe.br

cristino@deinfo.ufrpe.br

andreaap@,click21.com.br

Christian D.A. Daniel

Centro de Informática (CIn)

Universidade Federal de Pernambuco (UFPE)

Recife - PE, Brasil

chr.diego@gmail.com

* Corresponding author / autor para quem as correspondências devem ser encaminhadas

Recebido em 11/2007; aceito em 11/2009 após 1 revisão

Received November 2007; accepted November 2009 after one revision

\section{Resumo}

Os sistemas de geração e transmissão de energia elétrica (assim como outros sistemas complexos) podem ser modelados a partir de um grafo. Há necessidade de se otimizar recursos destinados aos sistemas de proteção de tais redes, assim como, a minimização de perdas advindas de grandes acidentes. A chamada avaliação probabilística de risco indica fragilidades no sistema em termos de proteção instalada e de fatores inerentes aos componentes. $\mathrm{O}$ artigo apresenta um modelo de análise dos riscos do sistema elétrico, atribuindo distribuições de falhas para cada componente ou conjunto de componentes, além de propor o modelo de propagação de tais falhas.

Palavras-chave: avaliação de risco; falhas em equipamentos; redes elétricas.

\begin{abstract}
The power generation and transmission systems (as well as other complex systems) can be represented from a graph. It is necessary to optimize resources regarded to the protection systems of such networks, as well as, the minimization of happened losses of great accidents. The so called probabilistic risk assessment indicates fragilities in the system in terms of installed protection and inherent factors to the components. This paper presents a model of analysis of the risks of the power system, attributing distributions of imperfections to each component or set of components, beyond considering the model of propagation of such imperfections.
\end{abstract}

Keywords: risk assessment; equipment failures; electric networks. 


\section{Introdução}

Para o estudo de grandes sistemas de equipamentos concatenados utiliza-se, geralmente, uma estrutura combinatória de grafos, sobre os quais pode-se definir funções que determinam um certo fluxo nesta estrutura. Denomina-se rede a este grafo em que está definido o fluxo. Especificamente, o sistema elétrico de geração e transmissão de energia elétrica pode ser modelado desta maneira. O principal objetivo deste trabalho é mostrar como se pode mapear o risco em um sistema elétrico, ou seja, uma avaliação da probabilidade de um acidente no sistema, cujas consequências acarretem custos financeiros, de vidas humanas ou custos ambientais. Aqui estarão apresentados resultados preliminares da avaliação de risco, estudo de confiabilidade e modelagem para o sistema de geração e transmissão da Cia Hidro Elétrica do São Francisco - CHESF.

\section{Representações para o sistema elétrico}

O sistema de geração e transmissão de energia elétrica possui uma considerável quantidade de componentes, distribuídos em subestações e plantas de geração, conforme um planejamento estratégico para sua distribuição ao consumidor final. Tais equipamentos e suas conexões podem ser representados por um grafo $G=(V, E)$, para o qual o conjunto de vértices corresponde ao conjunto de equipamentos e $E$, o conjunto de arestas, representa as ligações entre os equipamentos.

Estes componentes pertencem às seguintes classes: $(\mathrm{G})$ Geradores; $(\mathrm{T})$ Transformadores e Autotrafos; (R) Reatores (em série e "shunt"); (B) Barramentos; (CS) Chaves Seccionadoras; (L) Linhas de Transmissão; (C) Compensadores (Estáticos e Síncronos); (CP) Capacitores (em série e "shunt"); (D) Disjuntores, além dos equipamentos do sistema de proteção do sistema: relés (diversos tipos), transformadores de corrente e de potencial, pára-raios, bobinas de bloqueio e enlace de fibra ótica.

A modelagem para a avaliação de risco não considera o modelo da rede elétrica como descrito acima, pois há uma necessidade de analisar os equipamentos individualmente e não os pontos elétricos (de mesmo potencial).

Assim, o modelo para a rede elétrica é dado por um grafo definido como se segue: seja $G$ um grafo cujo conjunto de vértices representa cada componente do sistema elétrico, e dois vértices são ligados por uma aresta se os componentes representados possuem ligação física ou lógica (ou seja, se o funcionamento/operação de um influencia no funcionamento/ operação do outro). Agora, considere a seguinte operação: retire todos os vértices que representam os disjuntores (equipamento de proteção que interrompe o fluxo de carga até o equipamento, ou conjunto de equipamentos que este esteja protegendo). As componentes conexas deste subgrafo serão denominadas setores da rede. Constrói-se um novo grafo: os vértices são os setores do subgrafo definido anteriormente e as arestas correspondem aos disjuntores que separam dois setores. Tal grafo é denominado grafo de isolamento.

Estaremos interessados em calcular o risco associado a cada equipamento nos diversos setores da rede elétrica, definidos pelo grafo de isolamento. 


\section{Enumeração de estados}

Seja $C=\left\{c_{1}, c_{2}, \ldots, c_{n}\right\}$ o conjunto de componentes do sistema elétrico. Cada componente pode estar em dois estados: ligado e desligado. Isso é representado pela seguinte função

$$
s\left(c_{i}\right)=s_{i}=\left\{\begin{array}{cc}
0, & \text { se } c_{i} \text { está no estado desligado; } \\
1, & \text { caso contrário }
\end{array}\right.
$$

Denota-se por $s=\left(s_{1}, s_{2}, \ldots, s_{n}\right)$ a $n$-upla que representa um estado do sistema. Se $P_{i}$ e $Q_{i}=1-P_{i}$ (Cristino et al., 2007 e Cordeiro et al., 2007) denotam as probabilidades de sucesso (estado ligado) e falha do $i$-ésimo componente, a probabilidade de um estado do sistema é

$$
P(s)=\prod_{i=1}^{n_{f}} Q_{i} \prod_{j=1}^{n-n_{f}} P_{j},
$$

em que $n_{f}$ é o número de componentes falhados (ou nos estados desligado). Se todos os componentes estão ligados então $n_{f}=0$ e, define-se naturalmente,

$$
P(s)=\prod_{i=1}^{n} P_{i}
$$

No caso de descrição do estado do sistema, tem-se que

$$
f(s)=P(s) \sum_{k=1}^{n} \alpha_{k}, \quad \text { e } d(s)=\frac{1}{\sum \alpha_{k}}
$$

em que $f(s)$ denota a frequência de um estado e $d(s)$ é a duração média de tal estado. Tem-se

$$
\alpha_{k}= \begin{cases}\lambda_{k}, & \text { taxa de falha; } \\ \mu_{k}, & \text { taxa de reparo, }\end{cases}
$$

usado de forma conveniente na transição de estados $s$. Se denotarmos por $S$ o conjunto de todos os estados, então, a probabilidade acumulada de falha do sistema é

$$
P_{f}=\sum_{s \in S} P(s)
$$

e a frequência de falha do sistema reduz-se a

$$
F_{f}=\sum_{s \in S} f(s)-\sum_{n, m \in S} f_{n m},
$$

em que $f_{n m}$ é a frequência de transição do estado $n$ para o estado $m$. Assim, é natural obtermos a duração acumulada da frequência de falha do sistema como

$$
D_{f}=\frac{P_{f}}{F_{f}} .
$$




\section{Funções usadas na análise do sistema}

Em todos os casos, para cada estado de falha do sistema, funções de risco podem ser obtidas através de técnicas de análise do sistema. Por exemplo, se for considerado uma função de diminuição de carga, $C(s)$, devido ao estado do sistema $s$, tem-se que

$$
E(C)=\sum_{s \in S} C(s) P(s)
$$

é a expectativa desta função para todos os estados de falha do sistema.

Os três métodos de análise do risco de falha têm como principal parâmetro a indisponibilidade de energia provocada por uma configuração (estado) particular do sistema. Além da configuração determinada pelo sequenciamento dos diversos componentes, exigências especiais de carga do sistema podem ser consideradas.

Na próxima seção, há uma nova definição da probabilidade de falha de um componente, que leva em conta a configuração (topologia) do sistema. Tal definição torna possível a definição do risco de falha, que será utilizada na classificação dos equipamentos do sistema elétrico.

\section{Definição de risco}

Seja $C=\left\{c_{1}, c_{2}, \ldots, c_{n}\right\}$ o conjunto de componentes. Seja $G=(C, E)$, o grafo de isolamento (dirigido) tal que

$$
E=\left\{e=\left[c_{i} c_{j}\right]: \text { falha/desligamento } c_{i} \Rightarrow \text { falha/desligamento } c_{j}\right\} .
$$

Associados a cada vértice (componente) de $G$ existem dois eventos (locais):

- $c_{i}$ está no estado $u$ (upper, ligado), ou $c_{i}$ é um $u$-vértice, ou

- $c_{i}$ está no estado $d$ (down, desligado), ou $c_{i}$ é um $d$-vértice.

Estes eventos são considerados complementares, ou seja, um é a negação do outro. Aqui serão denotados por $u_{c_{i}}$ e $d_{c_{i}}$, respectivamente. Eventos mais detalhados são da forma $u^{C_{1}} d^{C_{2}}$ com $C_{1} \cup C_{2}=C$ e $C_{1} \cap C_{2}=\varnothing$. Estes são chamados eventos elementares. $\mathrm{O}$ conjunto de todos os eventos elementares é chamado espaço de eventos, que é denotado por $\Omega$. A soma (lógica) de dois eventos $a$ e $a^{\prime}$ é denotada por $a+a^{\prime}$. Obviamente, $u_{c_{i}}+d_{c_{i}}=1$. Dois eventos $a$ e $a^{\prime}$ são chamados incompativeis ou disjuntos se $a+a^{\prime}=0$.

Eventos formados por somas finitas de eventos-produto finitos são chamados eventos locais. Os eventos formados pelo fecho da coleção de eventos locais sobre somas enumeráveis e produtos enumeráveis são chamados eventos aleatórios.

Eventos mais gerais são obtidos tomando-se o fecho da coleção de eventos aleatórios sobre produtos e somas arbitrárias. Considerando-se a completa distributividade, cada evento pode ser escrito unicamente como uma soma de eventos elementares, logo existe uma correspondência 1-a-1 entre eventos e subconjuntos do espaço de eventos.

Defina $P_{i}=P\left(c_{i}\right)$ a probabilidade de eventos locais sobre o vértice $c_{i}$ ser um $d$-vértice ou um $u$-vértice, independente de todos os outros componentes. Temos que: 
1. $P(0)=0$ e $P(1)=1$.

2. $P\left(d_{c_{i}}\right)=p_{i}$ e $P\left(u_{c_{i}}\right)=q_{i}=1-p_{i}, 0 \leq p_{i} \leq 1, i=1, \ldots, n$.

3. Para produtos finitos $u^{C^{\prime}} d^{C^{\prime \prime}}$ com $C^{\prime} \cap C^{\prime \prime}=\varnothing, P\left(u^{C^{\prime}} d^{C^{\prime \prime}}\right)=p^{C^{\prime}} q^{C^{\prime \prime}}$, ou seja, os eventos são considerados sendo independentes.

4. Para somas finitas de eventos-produto finitos tem-se: $P\left(\sum_{i=1}^{n} a_{i}\right)=\sum_{i=1}^{n} P\left(a_{i}\right)$.

Denominamos $P$ como a probabilidade primária de desligamento ou falha. Seja

$$
\operatorname{custo}\left(c_{i}\right)=\text { custo desligamento/falha de } c_{i} \text {. }
$$

A função custo $\left(c_{i}\right)$, para $c_{i} \in C$, é uma função real não-negativa dada em termos absolutos do custo de permanência de um componente no estado $d$, ou pode ser normalizada pelo "maior" custo de falha/desligamento. Tal função é obtida levando-se em conta os contratos de serviço, multas previstas, aliados ao custo de manutenção e substituição de componentes.

Defina, agora, um acidente em $G$ como sendo um par

$$
a=\left(v,\left\{e_{1}, \ldots, e_{k}\right\}\right),
$$

em que $v$ é um vértice de $G$, que representa um setor apresentando uma falha e $\left\{e_{1}, \ldots, e_{k}\right\}$ é o conjunto de arestas (representando os disjuntores no grafo de isolamento), que não estão em funcionamento, ou seja, que por problemas internos ou do sistema de proteção, tais disjuntores não são acionados adequadamente, não interrompendo o fluxo de carga. Além disso, o subgrafo de $G$ induzido por $\left\{e_{1}, \ldots, e_{k}\right\}$ e contendo $v$ é conexo.

Para a definição do risco de um setor ainda devemos supor a seguinte hipótese adicional: para cada setor $v$ o surgimento de uma falha que possa ser considerada acidente é uma variável aleatória $\mathcal{A}_{v}$, que assume 1 , com probabilidade $P\left(d_{v}\right)=p_{v}$ e 0 , como probabilidade $1-p_{v}$. Ainda, cada aresta $e$ possui probabilidade $p_{e}$ de estar em estado de falha quando requerido.

Então, a probabilidade de ocorrência anual de um acidente é

$$
P(a)=p_{v} \times \prod_{i=1}^{k} p_{e_{i}} \times \prod_{e \notin a}\left(1-p_{e}\right) .
$$

Logo, o risco de ocorrência de um acidente $a$ é $R(a)=P(a) \times \operatorname{custo}(a)$. De maneira direta, temos que o risco de um determinado setor $v$ reduz-se a

$$
R(v)=\sum_{a=\left(v, E^{\prime}\right)} R(a) .
$$

\section{Risco sobre caminhos}

Uma outra abordagem de atribuição de risco pode ser dada com se segue: seja $G$ o grafo de isolamento, como descrito anteriormente. Note que $G$ é conexo. Se não o for, considere as definições a seguir para cada componente conexa. Para cada $v \in V(G)$, setores contendo um 
ou mais equipamentos, suponha que exista uma probabilidade $P(v)$ de surgimento de um sinal de queima. Tal sinal se propaga pela rede através de caminhos dirigidos a partir de $v$.

Cada aresta de $G$ é munida de um dispositivo (função) que, a partir do surgimento do sinal de queima em $v$, remove as arestas que saem de $v,\left(e_{1}=\left[v v_{1}\right], \ldots e_{k}=\left[v v_{k}\right]\right)$, com probabilidade $q\left(e_{i}\right)=q_{e_{i}}=1-p_{e_{i}}$. Seja custo $V(G) \rightarrow \mathfrak{R}^{+}$a função custo do vértice $v$, definida como antes. O risco de um vértice $v, R(v)$, pode ser calculada pela regra do produto como:

$$
\begin{aligned}
R(v)= & P(v)\left\{\operatorname{custo}(v) \prod_{x \in \Gamma(v)} q_{v x}+\sum_{u \in \Gamma(v)} p_{v u} \prod_{\substack{x \in \Gamma(v) \\
x \neq u}} q_{v x}(\operatorname{custo}(v)+\operatorname{custo}(u)) \prod_{\substack{y \in \Gamma(u) \\
y \neq v}} q_{u v}\right. \\
& +\sum_{u \in \Gamma(v)} \sum_{\substack{w \in \Gamma(u) \\
w \neq v}}\left(p_{\substack { u v \\
\begin{subarray}{c}{x \in \Gamma(v) \\
x \neq u{ u v \\
\begin{subarray} { c } { x \in \Gamma ( v ) \\
x \neq u } }\end{subarray}} q_{v x}\right)\left(p_{u w} \prod_{\substack{y \in \Gamma(u) \\
y \neq\{v, w\}}} q_{u y}\right)(\operatorname{custo}(v)+\operatorname{custo}(u)+\operatorname{custo}(w)) \prod_{\substack{z \in \Gamma(w) \\
z \neq u}} q_{w z} \\
& + \text { falhas por 3-caminhos....\}. }
\end{aligned}
$$

Na equação acima, $\Gamma(v)$ representa o conjunto de vizinhos de $v$ em $G$, ou seja, os vértices adjacentes a $v$.

\section{Avaliação do risco de um sistema}

Um sistema de potência consiste em muitas entidades. Desligamentos estocásticos de tais entidades formam as principais causas dos processos de falha do sistema. A primeira tarefa na avaliação do risco de um sistema é determinar modelos de desligamentos de seus componentes. As falhas de uma entidade (transformador, disjuntor, linha de transmissão, etc.) de um sistema elétrico são classificadas em duas categorias: desligamento independente e dependente. Cada categoria pode ainda ser classificada de acordo com os modos de desligamento. Na maioria dos casos, só são considerados desligamentos forçados reparáveis, considerando que (em alguns casos) são, também, modelados desligamentos planejados. Falhas devido ao envelhecimento dos componentes, também, são incorporadas na avaliação do risco tradicional. Há dois métodos básicos para selecionar os estados de um sistema elétrico: enumeração de estados e Simulação Monte Carlo. Ambos os métodos têm prós e contras. Em geral, as condições operacionais complexas não são consideradas e as probabilidades de falha dos seus componentes são bastante reduzidas, sendo que a técnica de enumeração de estados é mais eficiente. Quando as condições operacionais são complexas e envolvem um número grande de eventos, os métodos do tipo Monte Carlo são preferíveis.

A avaliação do risco de um sistema de potência é geralmente associada com as seguintes etapas: (1) determinar modelos de desligamento de componentes; (2) selecionar estados de sistemas e calcular as probabilidades deles estarem em funcionamento; (3) avaliar as consequências dos estados selecionados do sistema; (4) calcular índices de risco.

Como mencionado anteriormente, o risco é uma combinação de probabilidade e consequência. Com a informação obtida na segunda e terceira etapas, pode ser criado um índice que represente verdadeiramente o risco do sistema. 
De acordo com a análise dos estados do sistema, a avaliação do risco de um sistema elétrico pode ser dividida com relação a dois aspectos básicos: suficiência e segurança do sistema. A suficiência relaciona a existência de instalações suficientes dentro do sistema para satisfazer a demanda de carga dos consumidores e aos desligamentos operacionais dos componentes do sistema. A suficiência é, então, associada com as condições estáticas que não incluem processos dinâmicos. A confiabilidade relaciona a habilidade do sistema em responder às perturbações dinâmicas que surgem dentro do sistema. A confiabilidade é, então, associada com a resposta do sistema para quaisquer perturbações a que ele esteja sujeito. A maioria das técnicas de avaliação de risco que foram usadas nas aplicações a sistemas elétricos está no domínio da avaliação de suficiência.

Um sistema de potência inclui as três funções fundamentais de geração, transmissão (inclusive subestação) e distribuição. Tradicionalmente, as três zonas funcionais são incluídas em uma mesma função de utilidade. Estas três zonas funcionais podem ser tratadas separadamente para formar a geração independente, transmissão e companhias de distribuição. A avaliação de risco para um sistema global, inclusive geração, transmissão e distribuição, não é prático porque tal sistema é muito grande para controlar a capacidade de computação existente e as exigências de precisão. Por um lado, os algoritmos que modelam o risco são bastante diferentes para avaliar o risco de geração, transmissão, subestação e do sistema de distribuição.

Geralmente, torna-se necessário avaliar os benefícios relativos de alternativas diferentes, inclusive a opção de não fazer nada. O nível de necessidade das análises não é mais complexo que aquele que habilita os méritos relativos a serem avaliados. A habilidade para incluir um alto grau de precisão em cálculos nunca deveria anular a incerteza inerente aos dados. Um índice de risco absoluto, embora seja um objetivo ideal, é virtualmente impossível de se avaliar.

A natureza dos dados deve refletir a necessidade da avaliação do risco. Os dados devem ser suficientes para assegurar que um método de avaliação possa ser aplicado, mas restritivo bastante para assegurar que aqueles dados desnecessários não precisam ser coletados. Para os modelos simples, os dados relacionam os dois processos principais do comportamento dos componentes, isto é, os processos de falha e de reparo. Para modelos mais complexos, os dados estão associados com as taxas de transição entre os vários estados. A qualidade dos dados é um fator importante para ser considerado no processo de coleta dos dados.

O objetivo básico de um sistema de energia elétrica moderno é prover energia elétrica para seus clientes ao custo mais baixo possível e a um nível de risco aceitável. Há um conflito entre economia e risco. A avaliação do custo-risco deve ser uma aproximação apropriada na análise de riscos e dos fatores econômicos em um tratamento com base no valor monetário. $\mathrm{O}$ custo do risco de uma interrupção de energia por unidade de tempo deve ser avaliado bem como a energia esperada não provida. Entretanto, a quantificação do custo de interrupção é complexa. Deve ser computada a função de penalização de clientes que pode ser determinada em pesquisas de clientes durante vários anos.

Pode-se apresentar três métodos para avaliar o custo de interrupção de equipamentos:

- Método baseado em funções de penalização dos clientes: ela deve representar uma média por dano social aos clientes devida a interrupções de provisão de energia. É importante reconhecer que o custo de interrupção depende da região, país e sistema específico. 
- Método baseado em investimentos de capital: Há uma relação mensurável entre o capital investido e o índice de risco do sistema. Os custos de interrupção se baseiam nos investimentos de capital.

- Método baseado em produto doméstico total: O produto doméstico total para uma região pode ser obtido dividindo o custo estimado de perda de energia nesta região num ano pelo consumo total de energia elétrica anual correspondente. Este número reflete o dano econômico comum devido a $1 \mathrm{KWh}$ de perda de energia elétrica naquela região.

Na simulação de PRA são utilizados atualmente os métodos chamados Mininal Cutset (MCS) e diagramas de decisão binária (BDD) (Ahmad, 1988; Akers, 1978; Drechsler \& Becker, 1998; Füredi, Griggs \& Kleitman, 1989; Overstreet \& Yu, 2004).

\section{Simulação de cenários futuros}

Um dos mais desejáveis objetivos na análise de falhas de um equipamento é o seguinte: a partir do histórico de falhas do equipamento, produzir rapidamente milhões de cenários futuros cientificamente plausíveis do seu comportamento relativo a estas falhas. Para resolver esta questão, usando a hipótese de que a distribuição das falhas é exponencial (uma hipótese perfeitamente aceitável) definiu-se um autômato finito, ou seja, um grafo finito com pesos positivos nas arestas. Associamos a esse grafo um algoritmo muito simples capaz de simular com rapidez o comportamento futuro do equipamento com a mesma distribuição de falhas da que foi estimada a partir do histórico. Este autômato é um elemento básico para a simulação de situações mais complexas como as que aparecem em cálculo de risco no setor de geração e transmissão de energia.

A probabilidade de destruição do equipamento nobre é calculada simulando (bilhões de vezes) os cenários futuros para os fatores primitivos e lendo os vetores $0-1$ nas linhas verticais que cortam as linhas de tempo dos fatores primitivos. Cada linha vertical é um instante no tempo. Dada a probabilidade, o risco é obtido pelo seu produto com o custo da destruição. Em geral, a probabilidade é bem pequena e o custo bem alto. Assim, ordenar os riscos é um objetivo importante para tomar medidas preventivas. A partir das linhas de tempo, podem-se gerar os grafos que representam os estados (vértices) e suas transições (arestas), com pesos $\lambda_{i j}$ representando a taxa de transição do estado $i$ para o estado $j$. Usando variáveis aleatórias com distribuição exponencial com parâmetros $\lambda_{i j}^{(k)}$, tais parâmetros são obtidos por:

$$
\lambda_{i j}^{(k)}=\frac{n_{i j}^{(k)}}{\sum_{i} t_{i}}
$$

em que $n_{i j}^{(k)}$ é o número de passagens do estado $i$ para o estado $j$ relativo ao equipamento $k \mathrm{e}$ $\sum_{i} t_{i}$ é a soma dos intervalos rotulados por $i$, ou seja, o total dos tempos em que o equipamento permaneceu no estado $i$. De posse dos parâmetros $\lambda_{i j}^{(k)}$, passa-se ao algoritmo que define o autômato que expressa o comportamento futuro de um equipamento a partir de $N$, que representa o número de iterações, e do grafo com arestas com pesos positivos $\lambda_{i j}$. 
Tabela 1 - Algoritmo para o grafo de estados.

Comece no estado $i_{0} ; n=0$.

Estando em um estado $i$, sorteie para cada aresta $(i, j)$ um tempo $T_{i j}>0$ aleatório com distribuição exponencial de parâmetro $\lambda_{i j}^{(k)}$. Permaneça $T_{i j}$ no estado $i$ e vá para o estado $j$, tal que $T_{i j}=\min \left\{T_{i j^{\prime}}:\left(i, j^{\prime}\right)\right.$ é aresta $\} ; n=n+1$.

Se $n<N+1$, então volte para 2 , senão termine.

A saída do algoritmo é a linha de tempo com falhas para o equipamento em questão.

\subsection{Autômato e histórico de falhas}

De maneira geral, pode-se através da história de falhas/reparos de um dado equipamento obter um autômato que simula o futuro deste.

Na Figura 2, é denotado por $T_{i}$ o tempo de permanência do equipamento no estado $i$. Se $T_{i j}$ denotar o tempo de permanência do equipamento no estado $i$ até passar para o estado $j$, supondo que tem distribuição exponencial com parâmetros $\lambda_{i j}$, tem-se que $T_{i j} \sim E\left(\lambda_{i j}\right)$. Neste caso, a função de distribuição acumulada para este modelo é:

$$
\operatorname{Pr}\left(T_{i j}<x\right)=1-\exp \left(-\lambda_{i j} x\right), \quad x>0,
$$

sendo a função densidade de probabilidade dada por: $f_{T_{i j}}(x)=\lambda_{i j} \exp \left(-\lambda_{i j} x\right), \mathrm{x}>0$.

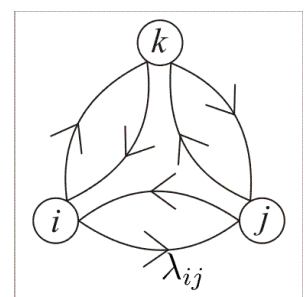

Figura 1 - Exemplo de grafo de falha.



Figura 2 - Exemplo de linha de tempo.

O tempo de permanência do equipamento no estado $i$ é $T_{i}=\min _{j \neq i}\left\{T_{i j}\right\}$. Da função de distribuição acumulada da distribuição exponencial, tem-se $T_{i} \sim E\left(\lambda_{i}\right)$, em que $\lambda_{i}=\sum_{j \neq i} \lambda_{i j}$. 
Para a estimação no longo horizonte $T$, podemos calcular a média dos $T_{i}$ por $\bar{T}_{i}=\frac{\sum T_{i}}{n_{i}}$ e, portanto, $\hat{\lambda}_{i}=\frac{1}{\bar{T}_{i}}=\frac{n_{i}}{\sum T_{i}}$ representa a taxa de permanência no estado $i$.

Se $P_{i}$ é a probabilidade assintótica do equipamento estar no estado $i$, a frequência de visitas do estado $i$ para o estado $j$ é dada por $f_{i j}=P_{i} \lambda_{i j}$. Logo, para a estimação no longo horizonte $T$ :

$$
\hat{P}_{i}=\frac{\sum T_{i}}{T}, \quad f_{i j}=\frac{n_{i j}}{T} .
$$

Então,

$$
\hat{\lambda}_{i j}=\frac{n_{i j}}{T} \frac{T}{\sum T_{i}}=\frac{n_{i j}}{\sum T_{i}}
$$

A frequência de transições do estado $i, f_{i}$, é dada por $f_{i}=P_{i} \sum_{j \neq i} \lambda_{i j}$.

E a estimação no longo horizonte $T$ é $\hat{f}_{i}=\frac{\sum T_{i}}{T}=\frac{\sum_{j \neq i} n_{i j}}{T_{i}}$. O número de transições $N_{i}(t)$ do estado $i$ no tempo $t$ pode ser tratado como um processo de Poisson de parâmetro $P_{i} \lambda_{i} t$, ou seja, $N_{i}(t) \sim P\left(P_{i} \lambda_{i} t\right)$, em que $\lambda_{i}=\sum_{j \neq i} \lambda_{i j}$. Agora, se $N_{i j}(t)$ é o número de transições do estado $i$ para o estado $j$ no tempo $t$, tem-se que

$$
N_{i j}(t) \sim P\left(P_{i} \lambda_{i j} t\right),
$$

A probabilidade de $r$ transições do estado $i$ para o estado $j$ no tempo $t$, para $r=0,1,2, \ldots$, é dada por

$$
\begin{aligned}
& \operatorname{Pr}\left\{N_{i j}(t)=r\right\}=(r !)^{-1} e^{-P_{i} \lambda_{i j} t}\left(P_{i} \lambda_{i j} t\right)^{r}, \\
& \operatorname{Pr}\left\{N_{i j}(t)>r\right\}=1-\operatorname{Pr}\left\{\chi_{2(r+1)}^{2}>2 P_{i} \lambda_{i j} t\right\} .
\end{aligned}
$$

No caso específico deste projeto, para sua otimização, alguns pontos foram considerados críticos no algoritmo. $\mathrm{O}$ autômato designado para representar a vida de um equipamento $k$ é representado por um grafo dirigido com pesos nas arestas (como especificado nessa subseção). O peso das arestas representa $\lambda_{i j}^{(k)}$, a taxa de transição do estado $i$ para o estado $j$. Na computação do problema a fim de se otimizar o tempo de acesso ao grafo e para melhorar a sua representação, foi escolhida uma implementação utilizando triplas ordenadas. Esse conjunto precisa receber apenas um par-ordenado $(i, j)$ para recuperar todos os $\lambda_{i j}^{(k)}$. Para um dado estado inicial $i$, também é possível obter todos os parâmetros com apenas este índice. 


\section{Modelagem do tempo de funcionamento de um transformador}

O tempo de funcionamento de qualquer equipamento do sistema elétrico definido como o tempo de funcionamento até o primeiro acidente (ou falha) é modelado através de um modelo exponencial log-linear de regressão. Esse modelo considera que a distribuição do tempo segue a distribuição exponencial cujo logaritmo da média é dado por uma estrutura linear contendo as variáveis que explicam o comportamento desse tempo e parâmetros associados desconhecidos. Nessa seção, nos restringiremos aos transformadores do sistema CHESF.

Neste trabalho, foram utilizados os dados do tempo de funcionamento em meses de 61 transformadores e as suas respectivas características físicas. O objetivo é estimar uma equação de regressão para modelar a média do tempo de funcionamento (tempo) dos transformadores, considerando como variáveis explicativas a espécie (especie) do equipamento, o número de enrolamentos (enrolam), o tipo do transformador (tipo) e a sua potência (em MVA) (pot). Ilustraremos aqui a modelagem do tempo de funcionamento de um transformador, mas este método pode ser aplicado para qualquer equipamento.

As variáveis tempo e pot são quantitativas e especie, enrolam e tipo são variáveis qualitativas, também, denominadas fatores. $\mathrm{O}$ fator especie tem dois níveis, monofásico $(\mathrm{M})$ e trifásico (T); o fator enrolam tem três níveis, 1 (A), 2 (B) e 3 (C) correspondentes a um, dois e três, rolamentos, respectivamente; e, finalmente, o fator tipo, também, com três níveis, transformador (A), reator (B) e trafo elevador (C). A potência (pot) é uma covariável contínua medida em MVA e o tempo (tempo) representa o número de meses ocorridos entre a energização e a falha detectada em um equipamento.

Supondo que o tempo de funcionamento segue a distribuição exponencial e considerando uma função de ligação logarítmica para a média dessa distribuição, um modelo que melhor se ajusta aos dados é dado pela seguinte equação obtida através do software $R$ :

$\log \hat{\mu}=5.3556-1.3078$ enrolamB -2.2298 enrolam $C+1.9833$ tipoC -0.0089 pot

+1.6683 especieT $\times$ enrolamB +2.6022 especieT $\times$ enrolam $C-1.9019$ especie $T \times$ tipo $C$.

Essa equação pode, também, ser escrita como:

$$
\begin{gathered}
\hat{\mu}=211.7913 \times 0.2704^{\text {enrolam } B} \times 0.1075^{\text {enrolam } C} \times 7.2667^{\text {tipoC }} \times 0.9912^{\text {pot }} \times \\
5.3032^{\text {especieT } \times \text { enrolam } B} \times 13.4934^{\text {especieT } \times \text { enrolam } C} \times 0.1493^{\text {especieT } \times \text { tipoC }} .
\end{gathered}
$$

Este modelo foi aceito pelo teste do desvio, pois possui desvio (21.097) inferior ao ponto crítico (ou seja, valor tabelado) da distribuição qui-quadrado com 53 graus de liberdade ao nível de 5\% (70.99). As principais estimativas dos parâmetros são significativas, com exceção dos parâmetros enrolamB, especieT:tipoC.

Adota-se aqui os resíduos e interação de Anscombe deduzidos para o modelo exponencial, com expressão

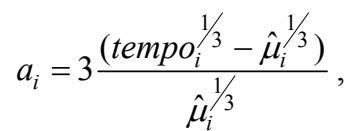

pois esses resíduos podem ser considerados, aproximadamente, normais.

O gráfico de $a_{i}$ versus $\hat{\mu}_{i}$, mostrado na Figura 3, não revela dados aberrantes (nenhum resíduo fora do intervalo $(-2,2))$ e os pontos se apresentam aleatoriamente distribuídos, sem nenhum padrão definido, concluindo-se, então, que esses tempos são variáveis independentes. 
A Figura 4 apresenta os valores observados versus os valores ajustados, mostrando alguma dispersão em torno da primeira bissetriz. A Figura 5 apresenta o gráfico da variável dependente modificada estimada $z$ versus o preditor linear estimado $\hat{\eta}$ indicando que a função de ligação logarítmica está correta.

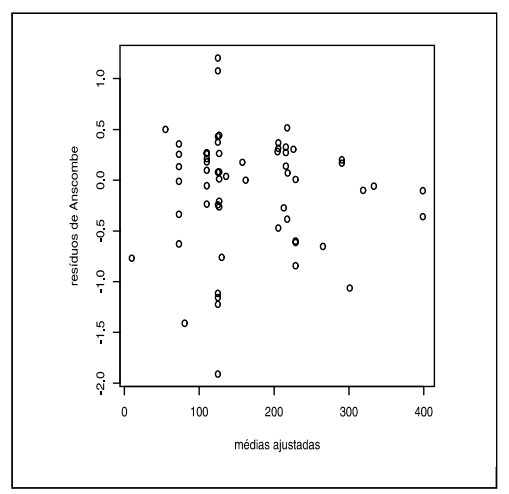

Figura 3 - Resíduos de Anscombe versus médias ajustadas.

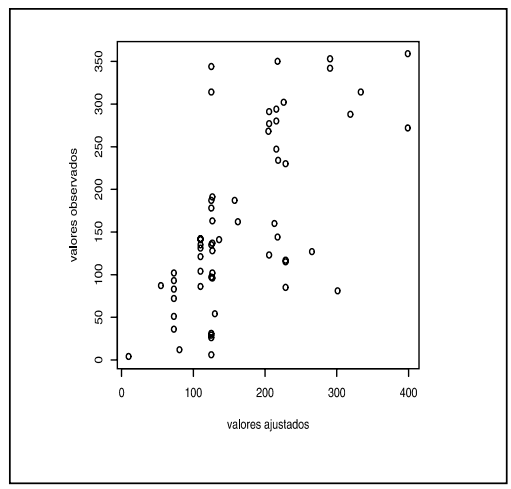

Figura 4 - Valores observados versus valores ajustados.

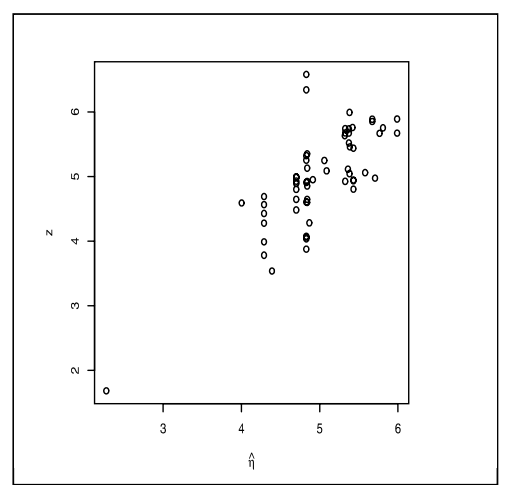

Figura 5 - Gráfico de $z$ versus $\hat{\eta}$. 
Os resíduos ordenados versus os quantis da $N(0,1)$ mostrados na Figura 6, suportam a distribuição exponencial para tempo.

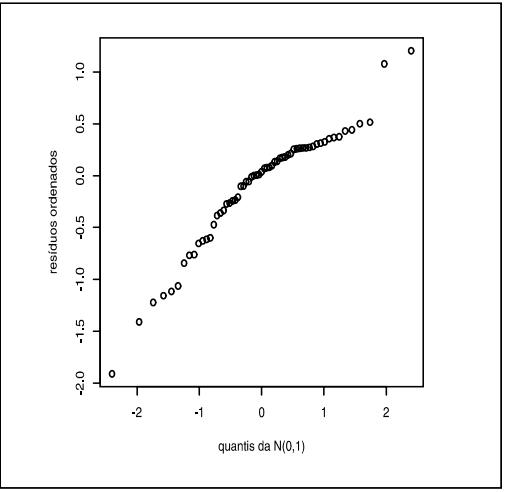

Figura 6 - Resíduos de Anscombe ordenados versus os quantis da $\mathrm{N}(0,1)$.

A Figura 7 apresenta o gráfico da estatística de Cook versus o índice das observações. Nota-se que as observações \#45 e \#49 são pontos influentes.

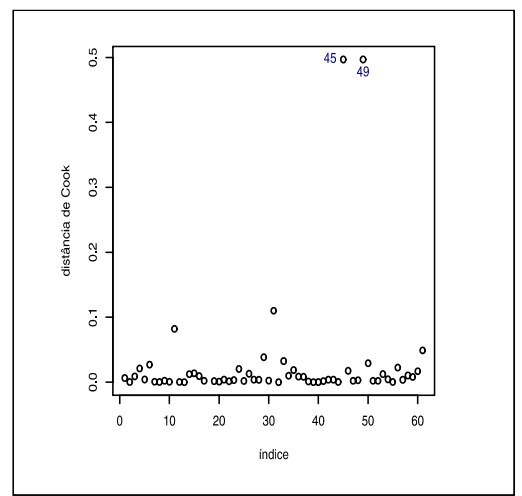

Figura 7 - Estatística de Cook versus o índice das observações.

Duas das observações que foram detectadas como pontos influentes no modelo se referem aos componentes do tipo transformador monofásico com dois enrolamentos. Como existem apenas estas duas observações com estas características elas foram mantidas no modelo. A chance do transformador só sofrer acidente após o tempo $t$ (sobrevida ao tempo $t$ ) foi calculada por:

$$
R(t)=\exp \left\{-t \exp \left(-x^{T} \beta\right\}=\exp (-t \hat{\mu}) .\right.
$$

As Tabelas 2 e 3 apresentam as confiabilidades dos transformadores para as espécies monofásicos e trifásicos, respectivamente. As curvas de sobrevidas dessas duas espécies de transformadores por tipo de equipamento estão dadas nas Figuras 8 (monofásicos) e 9 (trifásicos). Pode-se concluir que para os equipamentos monofásicos a sobrevida é maior 
para o tipo trafo-elevador, depois reator e bem menor para o transformador. Entretanto, para os equipamentos trifásicos, a sobrevida é superior para o tipo reator, depois trafo-elevador e inferior quando o equipamento é um transformador.

Tabela 2 - Confiabilidade dos transformadores monofásicos.

\begin{tabular}{cccc}
\hline Meses & $\begin{array}{c}\text { Transformador } \\
\text { 02T1-1-CRD }\end{array}$ & $\begin{array}{c}\text { Reator } \\
\text { 05E2-B-OLD }\end{array}$ & $\begin{array}{c}\text { Trafo Elevador } \\
\text { 01T3-A-USD }\end{array}$ \\
\hline 12 & 0.8033 & 0.9155 & 0.9646 \\
24 & 0.6453 & 0.8381 & 0.9305 \\
36 & 0.5183 & 0.7673 & 0.8976 \\
48 & 0.4164 & 0.7024 & 0.8659 \\
60 & 0.3345 & 0.6430 & 0.8353 \\
120 & 0.1119 & 0.4135 & 0.6977 \\
180 & 0.0374 & 0.2659 & 0.5828 \\
240 & 0.0125 & 0.1710 & 0.4868 \\
\hline
\end{tabular}

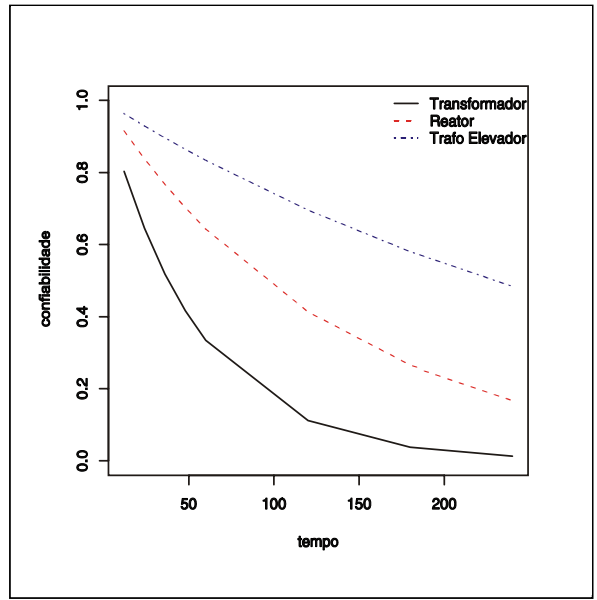

Figura 8 - Curva de sobrevida a acidentes para equipamentos monofásicos.

Tabela 3 - Confiabilidade dos transformadores trifásicos.

$\begin{array}{cccc}\text { Meses } & \begin{array}{c}\text { Transformador } \\ \text { 04T2-GNN }\end{array} & \begin{array}{c}\text { Reator } \\ \text { 02A1-SMD }\end{array} & \begin{array}{c}\text { Trafo Elevador } \\ \text { 01T1-UTC }\end{array} \\ 12 & 0.9095 & 0.9558 & 0.9286 \\ 24 & 0.8272 & 0.9135 & 0.8623 \\ 36 & 0.7524 & 0.8731 & 0.8007 \\ 48 & 0.6843 & 0.8345 & 0.7436 \\ 60 & 0.6224 & 0.7976 & 0.6905 \\ 120 & 0.3874 & 0.6361 & 0.4768 \\ 180 & 0.2411 & 0.5074 & 0.3292 \\ 240 & 0.1501 & 0.4047 & 0.2273\end{array}$




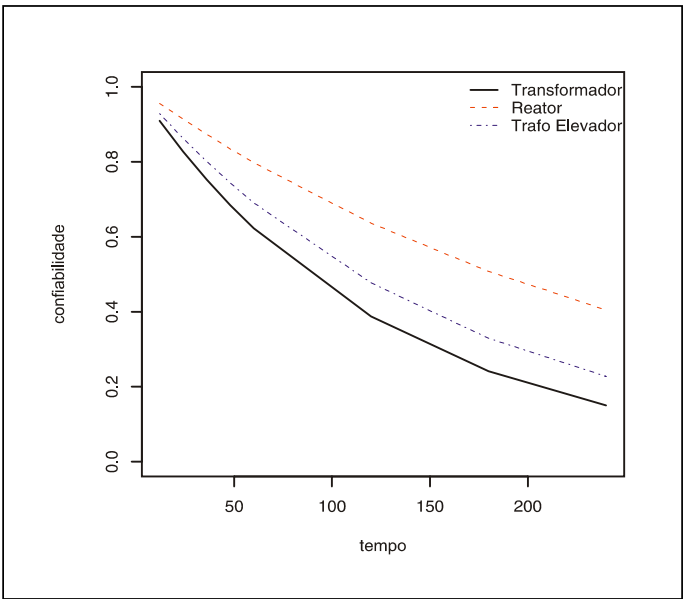

Figura 9 - Curva de sobrevida a acidentes para equipamentos trifásicos.

\section{Modelo com taxa de falha não constante}

Agora, supondo que o tempo de funcionamento segue a distribuição gama e considerando a mesma função de ligação do modelo anterior, ligação logarítmica, um modelo que melhor se ajusta aos dados é dado pela seguinte equação:

$$
\begin{aligned}
\log \hat{\mu}= & 5.5905-1.2617 \text { especieT }-1.1019 \text { enrolamB }-2.0134 \text { enrolam } C+1.4771 \text { tipo } C- \\
& 0.0152 \text { pot }+2.2977 \text { especieT } \times \text { enrolam } B+3.2462 \text { especieT } \times \text { enrolam } C- \\
& 1.9756 \text { especieT } \times \text { tipoC }+0.0090 \text { especie } T \times \text { pot }+0.0069 \text { tipo } \times \text { pot } .
\end{aligned}
$$

Essa equação pode, também, ser escrita como:

$$
\begin{aligned}
\hat{\mu}=267.8811 \times 0.2831^{\text {especieT }} \times 0.3322^{\text {enrolamB }} \times 0.1355^{\text {enrolam } C} \times 4.3804^{\text {tipoC }} \times \\
\quad 0.9849^{\text {pot }} \times 9.9510^{\text {especieT } \times \text { enrolamB }} \times 25.6930^{\text {especieT } \times \text { enrolam } C} \times 0.1387^{\text {especieT } \times \text { tipoC }} \times \\
\quad 1.0091^{\text {especieT } \times \text { pot }} \times 1.0070^{\text {tipoC } \times \text { pot }} .
\end{aligned}
$$

Este modelo também foi aceito pelo teste do desvio, pois possui desvio (19.348) inferior ao ponto crítico (ou seja, valor tabelado) da distribuição qui-quadrado com 50 graus de liberdade ao nível de 5\% (67.5048). As estimativas dos parâmetros são significativas.

A expressão dos resíduos de Anscombe para o modelo gama é a mesma deduzida para o modelo exponencial. Segundo o gráfico dos resíduos de Anscombe versus médias ajustadas mostrado na Figura 10 nenhuma observação excedeu o intervalo $(-2,2)$, portanto não existem dados aberrantes e os pontos se apresentam aleatoriamente distribuídos, sem nenhum padrão definido, concluindo-se, então, que esses tempos são variáveis independentes. 


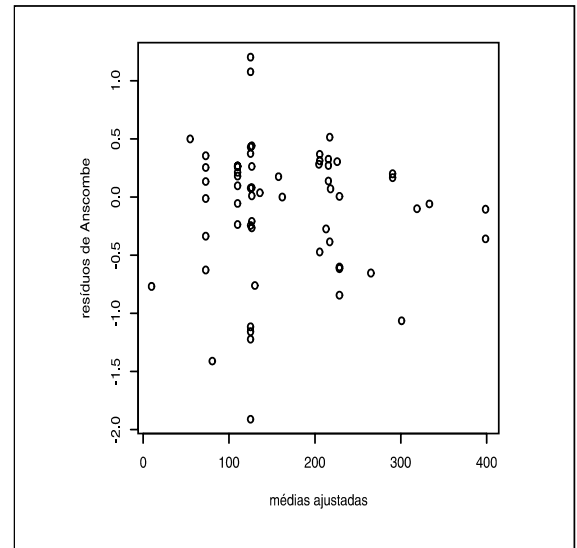

Figura 10 - Resíduos de Anscombe versus médias ajustadas.

A Figura 11 apresenta os valores observados versus os valores ajustados para o modelo gama, mostrando alguma dispersão em torno da primeira bissetriz. Alguns pontos foram melhor ajustados quando comparado ao modelo exponencial. Foi verificado que a função de ligação logarítmica também está correta para o modelo gama.

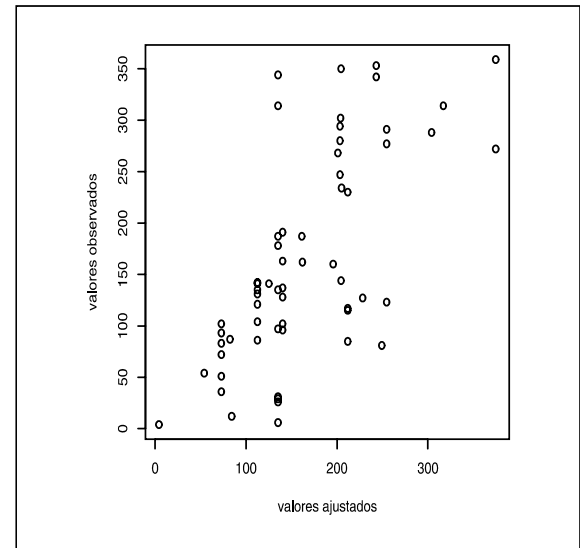

Figura 11 - Valores observados versus valores ajustados.

Os resíduos ordenados versus os quantis da $N(0,1)$ mostrados na Figura 12 suportam a distribuição gama para tempo. 


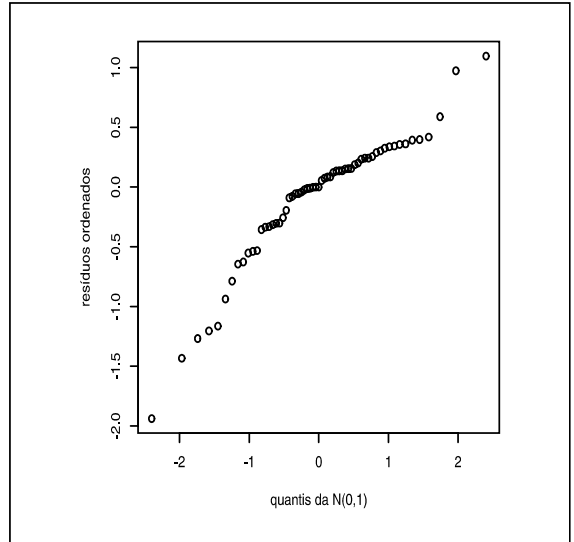

Figura 12 - Resíduos de Anscombe ordenados versus os quantis da N(0,1).

Para o modelo gama também foi detectada a presença de pontos influentes através da estatística de Cook. Esses pontos foram as mesmas observações, \#45 e \#49, encontradas para o modelo exponencial.

A chance do transformador só sofrer acidente após o tempo $t$ (sobrevida ao tempo $t$ ), modelo gama, foi calculada por:

$$
R(t)=\mathrm{P}(y \geq t)=1-\frac{1}{\Gamma(\hat{\phi})}\left(\frac{\hat{\phi}}{\hat{\mu}}\right) \int_{0}^{t} x^{\hat{\phi}^{\wedge}-1} \exp \left(\frac{-x \hat{\phi}}{\hat{\mu}}\right) d x .
$$

As Tabelas 4 e 5 apresentam as sobrevidas a acidentes dos transformadores para as espécies monofásicos e trifásicos, respectivamente. As curvas de sobrevidas dessas duas espécies de transformadores por tipo de equipamento estão dadas nas Figuras 13 (monofásicos) e 14 (trifásicos). O mesmo comportamento para os equipamentos monofásicos e trifásicos foi obtido em relação ao modelo anterior, sendo que para o modelo gama a sobrevida foi menor para todos os equipamentos, com exceção do tipo transformador que para o tempo maior que 120 meses a sobrevida aumentou.

Tabela 4 - Confiabilidade dos transformadores monofásicos.

\begin{tabular}{cccc}
\hline Meses & $\begin{array}{c}\text { Transformador } \\
\text { 02T1-1-CRD }\end{array}$ & $\begin{array}{c}\text { Reator } \\
\text { 05E2-B-OLD }\end{array}$ & $\begin{array}{c}\text { Trafo Elevador } \\
\text { 01T3-A-USD }\end{array}$ \\
\hline 12 & 0.9966 & 0.9992 & 1 \\
24 & 0.9671 & 0.9913 & 0.9997 \\
36 & 0.8959 & 0.9682 & 0.9986 \\
48 & 0.7891 & 0.9265 & 0.9961 \\
60 & 0.6635 & 0.8673 & 0.9916 \\
120 & 0.1701 & 0.4646 & 0.9292 \\
180 & 0.0270 & 0.1765 & 0.8005 \\
240 & 0.0033 & 0.0546 & 0.6372 \\
\hline
\end{tabular}




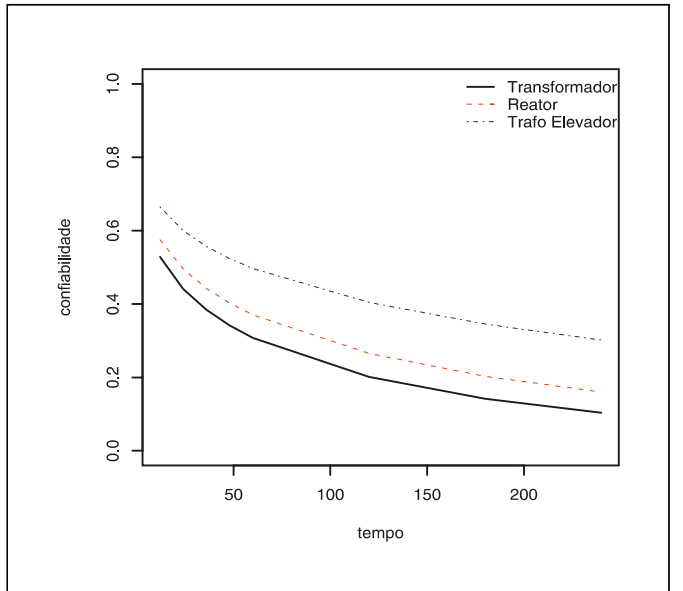

Figura 13 - Curva de confiabilidade para equipamentos monofásicos.

Tabela 5 - Confiabilidade dos transformadores trifásicos.

\begin{tabular}{cccc}
\hline Meses & $\begin{array}{c}\text { Transformador } \\
\text { 04T2-GNN }\end{array}$ & $\begin{array}{c}\text { Trafo Elevador } \\
\text { 02A1-SMD }\end{array}$ & $\begin{array}{c}\text { Reator } \\
\text { 01T1-UTC }\end{array}$ \\
\hline 12 & 0.9995 & 0.9999 & 0.9997 \\
24 & 0.9940 & 0.9989 & 0.9963 \\
36 & 0.9777 & 0.9955 & 0.9859 \\
60 & 0.9013 & 0.9759 & 0.9334 \\
120 & 0.5511 & 0.8336 & 0.6518 \\
180 & 0.2485 & 0.6093 & 0.3520 \\
240 & 0.0926 & 0.3938 & 0.1601 \\
\hline
\end{tabular}

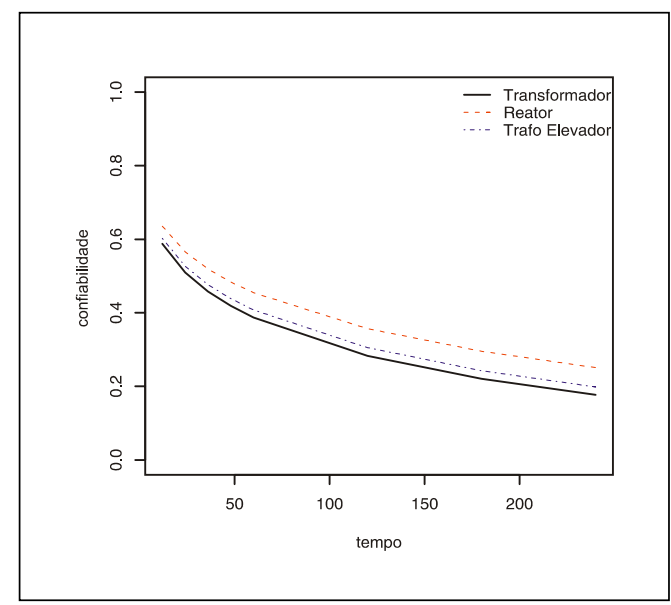

Figura 14 - Curva de confiabilidade para equipamentos trifásicos. 


\section{Conclusões}

A modelagem de sistemas de grande porte com o objetivo de análise de confiabilidade ou de risco de acidentes pode ser feita através dos grafos. Tais estruturas além de representar a topologia de uma rede, também, podem indicar a lógica de funcionamento, funções de controle do próprio sistema e a robustez das plantas. A partir dos históricos dos equipamentos é possível modelar as curvas de sobrevida de tais componentes e atribuir-lhes uma probabilidade de falha após um tempo $t$ arbitrário. A utilização de tais técnicas na avaliação de risco torna-se uma importante ferramenta na tomada de decisões por parte dos responsáveis de sistemas que podem ser modelados como apresentado.

\section{Agradecimentos}

Este projeto é financiado pela CHESF - Cia Hidro Elétrica do São Francisco, e ANEEL Agência Nacional de Energia Elétrica, através do contrato de Pesquisa e Desenvolvimento no. 443/06, ciclo 2002/2003.

\section{Referências Bibliográficas}

(1) Ahmad, S.H. (1988). Simple enumeration of minimal cutsets of acyclic directed graph. IEEE Transactions on Reliability, 37(5), 484-487.

(2) Akers, S.B. (1978). Binary Decision Diagrams. IEEE Transactions on Computers, 27(6).

(3) Billinton, R. \& Li, W. (1994). Reliability Assessment of Electric Power Systems Using Monte Carlo Methods. Plenum, New York.

(4) Cristino, C.T.; Cordeiro, G.M. \& Lins, S. (2007). Confiabilidade de Sistemas e Avaliação Probabilística de Riscos. Revista Brasileira de Matemática e Estatística, 25(2), 87-109.

(5) Cordeiro, G.M.; Cristino, C.T.; Lima, E.O.L. \& Melo, S.B. (2007). Modelos Markovianos, Percolação e Modelagem em Sistemas com Grande Número de Componentes. Revista Brasileira de Matemática e Estatística, 25(1), 99-116.

(6) Drechsler, R. \& Becker, B. (1998). Binary decision diagrams: theory and implementation. Kluwer Academic Publishers, Dordrecht.

(7) Füredi, Z.; Griggs, J.R. \& Kleitman, D.J. (1989). A Minimal Cutset of the Boolean Lattice with Almost all Members. Graphs and Combinatorics, 5(1), 327-332.

(8) Li, Wenyuan (2005). Risk Assessment of Power Systems - Models, Methods and Applications. IEEE Press, Piscataway.

(9) Overstreet, J. \& Yu, Y. (2004). A Simulation Method for Probabilistic Risk Assessment. In: Proceedings of Military Government and Aerospace Simulation Symposium.

(10) Sankarakrishnan, A. \& Billinton, A. (1996). Effective techniques for reliability worth assessment in composite power system networks using Monte Carlo simulation. IEEE Transactions on Power Systems, 11(3), 1255-1261.

(11) Schilling, M.T.; Souza, J.C.S de \& Coutto Filho, M.B. (2008). Power System Probabilistic Reliability Assessment: Current Procedures in Brazil. IEEE Transactions on Power Systems, 23(3), 868-876. 